\title{
Qualitative Properties and Numerical Solution of the Kolmogorov-Fisher Type Biological Population Task with Double Nonlinear Diffusion
}

\section{Dildora Kabulovna Muhamediyeva}

National University of Uzbekistan, Tashkent, Uzbekistan

Email: matematichka@inbox.ru

Received 24 July 2015; accepted 23 October 2015; published 26 October 2015

Copyright (C) 2015 by author and Scientific Research Publishing Inc.

This work is licensed under the Creative Commons Attribution International License (CC BY).

http://creativecommons.org/licenses/by/4.0/

(c) (i) Open Access

\section{Abstract}

In the present work we study the global solvability of the Kolmogorov-Fisher type biological population task with double nonlinear diffusion and qualitative properties of the solution of the task based on the self-similar analysis. In additional, in this paper we consider the model of two competing population with dual nonlinear cross-diffusion.

\section{Keywords}

Double Nonlinearity, Cross-Diffusion, Biological Population, A Parabolic System of Quasilinear Equations, Convective Heat Transfer, Numerical Solution, Iterative Process, Self-Similar Solutions

\section{Introduction}

Let's consider in the domain $Q=\{(t, x): 0<t, x \in R\}$ parabolic system of two quasilinear equations of reaction-diffusion with double nonlinear diffusion

$$
\left\{\begin{array}{c}
\frac{\partial u_{1}}{\partial t}=\frac{\partial}{\partial x}\left(D_{1} u_{2}^{m_{1}-1}\left|\frac{\partial u_{1}}{\partial x}\right|^{p-2} \frac{\partial u_{1}}{\partial x}\right)+k_{1}(t) u_{1}\left(1-u_{1}^{\beta_{1}}\right) \\
\frac{\partial u_{2}}{\partial t}=\frac{\partial}{\partial x}\left(D_{2} u_{1}^{m_{2}-1}\left|\frac{\partial u_{2}}{\partial x}\right|^{p-2} \frac{\partial u_{2}}{\partial x}\right)+k_{2}(t) u_{2}\left(1-u_{2}^{\beta_{2}}\right) \\
\left.u_{1}\right|_{t=0}=u_{10}(x),\left.\quad u_{2}\right|_{t=0}=u_{20}(x),
\end{array}\right.
$$

How to cite this paper: Muhamediyeva, D.K. (2015) Qualitative Properties and Numerical Solution of the KolmogorovFisher Type Biological Population Task with Double Nonlinear Diffusion. Journal of Applied Mathematics and Physics, 3, 1249 1255. http://dx.doi.org/10.4236/jamp.2015.310153 
which describes the process of biological populations of the Kolmogorov-Fisher in two-component nonlinear medium, the diffusion coefficient which is equal to $D_{1} u_{2}^{m_{2}-1}\left|\frac{\partial u_{1}}{\partial x}\right|^{p-2}, D_{2} u_{1}^{m_{2}-1}\left|\frac{\partial u_{2}}{\partial x}\right|^{p-2}$, where $m_{1}, m_{2}, p, \beta_{1}, \beta_{2}$ are positive real numbers, and $u_{1}=u_{1}(t, x) \geq 0, u_{2}=u_{2}(t, x) \geq 0$ are sought solution .

Below we investigate the qualitative properties of the considered problem by constructing self-similar system of equations for (1).

\section{Self-Similar System of Equations}

Self-similar system of equations we will construct by the method of nonlinear splitting [1]-[3].

Substitution in (1)

$$
u_{1}(t, x)=\mathrm{e}^{k_{1} t} v_{1}(t, x), \quad u_{2}(t, x)=\mathrm{e}^{k_{2} t} v_{2}(t, x)
$$

lead (1) to the form:

$$
\begin{aligned}
& \left\{\begin{array}{l}
\frac{\partial v_{1}}{\partial \tau_{1}}=\frac{\partial}{\partial x}\left(D_{1} v_{2}^{m_{1}-1}\left|\frac{\partial v_{1}}{\partial x}\right|^{p-2} \frac{\partial v_{1}}{\partial x}\right)+k_{1} \mathrm{e}^{\left[\left(\beta_{1}-p+2\right) k_{1}-\left(m_{1}-1\right) k_{2}\right] t} v_{1}^{\beta_{1}+1} \\
\frac{\partial v_{2}}{\partial \tau_{2}}=\frac{\partial}{\partial x}\left(D_{2} v_{1}^{m_{2}-1}\left|\frac{\partial v_{2}}{\partial x}\right|^{p-2} \frac{\partial v_{2}}{\partial x}\right)+k_{2} \mathrm{e}^{\left[\left(\beta_{2}-p+2\right) k_{2}-\left(m_{2}-1\right) k_{1}\right] t} v_{2}^{\beta_{2}+1}
\end{array}\right. \\
& \left.v_{1}\right|_{t=0}=v_{10}(\eta),\left.\quad v_{2}\right|_{t=0}=v_{20}(\eta) .
\end{aligned}
$$

Choosing

$$
\tau=\frac{\mathrm{e}^{\left[\left(m_{1}-1\right) k_{2}+(p-2) k_{1}\right] t}}{(p-2) k_{1}+\left(m_{1}-1\right) k_{2}}=\frac{\mathrm{e}^{\left[\left(m_{1}-1\right) k_{1}+(p-2) k_{2}\right] t}}{(p-2) k_{2}+\left(m_{2}-1\right) k_{1}},
$$

we get the following system of equations:

$$
\left\{\begin{array}{l}
\frac{\partial v_{1}}{\partial \tau}=\frac{\partial}{\partial x}\left(D_{1} v_{2}^{m_{1}-1}\left|\frac{\partial v_{1}}{\partial x}\right|^{p-2} \frac{\partial v_{1}}{\partial x}\right)-a_{1} \tau^{b_{1}} v_{1}^{\beta_{1}+1} \\
\frac{\partial v_{2}}{\partial \tau}=\frac{\partial}{\partial x}\left(D_{2} v_{1}^{m_{2}-1}\left|\frac{\partial v_{2}}{\partial x}\right|^{p-2} \frac{\partial v_{2}}{\partial x}\right)-a_{2} \tau^{b_{2}} v_{2}^{\beta_{2}+1}
\end{array}\right.
$$

where

$$
\begin{aligned}
& a_{1}=k_{1} \cdot\left[(p-2) k_{1}+\left(m_{1}-1\right) k_{2}\right]^{b_{1}}, \quad b_{1}=\frac{\left(\beta_{1}-p+2\right) k_{1}-\left(m_{1}-1\right) k_{2}}{(p-2) k_{1}+\left(m_{1}-1\right) k_{2}} \\
& a_{2}=k_{2} \cdot\left[(p-2) k_{2}+\left(m_{2}-1\right) k_{1}\right]^{b_{2}}, \quad b_{2}=\frac{\left(\beta_{2}-p+2\right) k_{2}-\left(m_{2}-1\right) k_{1}}{(p-2) k_{2}+\left(m_{2}-1\right) k_{1}}
\end{aligned}
$$

For the purpose of obtaining self-similar system for the system of Equation (3) we find first the solution of a system of ordinary differential equations [4]-[7]

$$
\left\{\begin{array}{l}
\frac{\mathrm{d} \bar{v}_{1}}{\mathrm{~d} \tau}=-a_{1} \tau^{b_{1}} \bar{v}_{1}^{\beta_{1}+1}, \\
\frac{\mathrm{d} \bar{v}_{2}}{\mathrm{~d} \tau}=-a_{2} \tau^{b_{2}} \bar{v}_{2}^{\beta_{2}+1},
\end{array}\right.
$$

in the form

$$
\bar{v}_{1}(\tau)=\tau^{-\alpha_{1}}, \quad \bar{v}_{2}(\tau)=\tau^{-\alpha_{2}},
$$

where 


$$
\alpha_{1}=\frac{b_{1}+1}{\beta_{1}}, \quad \alpha_{2}=\frac{b_{2}+1}{\beta_{2}} .
$$

And then the solution of system (3) is sought in the form

$$
v_{1}(t, x)=\bar{v}_{1}(t) w_{1}(\tau, x), v_{2}(t, x)=\bar{v}_{2}(t) w_{2}(\tau, x)
$$

and $\tau=\tau(t)$ is selected so

$$
\begin{aligned}
\tau(\tau) & =\int_{0}^{\tau} \bar{v}_{1}^{(p-2)}(t) \bar{v}_{2}^{\left(m_{1}-1\right)}(t) \mathrm{d} t=\int_{0}^{\tau} \bar{v}_{2}^{(p-2)}(t) \bar{v}_{1}^{\left(m_{2}-1\right)} \mathrm{d} t \\
& = \begin{cases}\frac{1}{1-\left[\alpha_{1}(p-2)+\alpha_{2}\left(m_{1}-1\right)\right]}(T+\tau)^{-\left[\alpha_{1}(p-2)+\alpha_{2}\left(m_{1}-1\right)\right]+1}, & \text { if } 1-\left[\alpha_{1}(p-2)+\alpha_{2}\left(m_{1}-1\right)\right] \neq 0, \\
\ln (T+\tau), & \text { if } 1-\left[\alpha_{1}(p-2)+\alpha_{2}\left(m_{1}-1\right)\right]=0,\end{cases}
\end{aligned}
$$

if $\alpha_{1}(p-2)+\alpha_{2}\left(m_{1}-1\right)=\alpha_{2}(p-2)+\alpha_{1}\left(m_{2}-1\right)$.

Then for $w_{i}(\tau, x), i=1,2$ we get the system of equations

$$
\left\{\begin{array}{l}
\frac{\partial w_{1}}{\partial \tau}=\frac{\partial}{\partial x}\left(D_{1} w_{2}^{m_{1}-1}\left|\frac{\partial w_{1}}{\partial x}\right|^{p-2} \frac{\partial w_{1}}{\partial x}\right)+\psi_{1}\left(w_{1}^{\beta_{1}+1}-w_{1}\right) \\
\frac{\partial w_{2}}{\partial \tau}=\frac{\partial}{\partial x}\left(D_{2} w_{1}^{m_{2}-1}\left|\frac{\partial w_{2}}{\partial x}\right|^{p-2} \frac{\partial w_{2}}{\partial x}\right)+\psi_{2}\left(w_{2}^{\beta_{2}+1}-w_{2}\right)
\end{array}\right.
$$

where

$$
\psi_{i}=\frac{1}{\left(1-\left[\alpha_{i}(p-2)+\alpha_{3-i}\left(m_{i}-1\right)\right]\right) \tau}, \quad i=1,2
$$

Consider the self-similar solution of system (5) of the form

$$
w_{1}(t, x)=f_{1}(\xi), w_{2}(t, x)=f_{2}(\xi), \quad \xi=|x| /(T+\tau)^{1 / p}
$$

Then substituting (7) into (5) with respect to $f_{1}(\xi), f_{2}(\xi)$ we get the following system of nonlinear degenerate self-similar equations:

$$
\left\{\begin{array}{l}
\frac{\mathrm{d}}{\mathrm{d} \xi}\left(f_{2}^{m_{1}-1}\left|\frac{\mathrm{d} f_{1}}{\mathrm{~d} \xi}\right|^{p-2} \frac{\mathrm{d} f_{1}}{\mathrm{~d} \xi}\right)+\frac{\xi}{p} \frac{\mathrm{d} f_{1}}{\mathrm{~d} \xi}+\theta_{1}\left(f_{1}-f_{1}^{\beta_{1}+1}\right)=0 \\
\frac{\mathrm{d}}{\mathrm{d} \xi}\left(f_{1}^{m_{2}-1}\left|\frac{\mathrm{d} f_{2}}{\mathrm{~d} \xi}\right|^{p-2} \frac{\mathrm{d} f_{2}}{\mathrm{~d} \xi}\right)+\frac{\xi}{p} \frac{\mathrm{d} f_{2}}{\mathrm{~d} \xi}+\theta_{2}\left(f_{2}-f_{2}^{\beta_{2}+1}\right)=0
\end{array}\right.
$$

where $\theta_{i}=\frac{1}{\left(1-\left[\alpha_{i}(p-2)+\alpha_{3-i}\left(m_{i}-1\right)\right]\right) \tau}, \quad i=1,2$. Let's build an upper solutions for system (8).

\section{Construction an Upper Solution}

If

$$
\beta_{i}=\left[(p-2)^{2}-\left(m_{1}-1\right)\left(m_{2}-1\right)\right] /\left[(p-1)\left(p-\left(m_{i}+1\right)\right)\right], \quad p>2+\sqrt{\left(m_{1}-1\right)\left(m_{2}-1\right)}, \quad i=1,2,
$$

Equation (8) has a local solution of the form

$$
\bar{f}_{1}(\xi)=A\left(a-\xi^{\gamma}\right)_{+}^{n_{1}}, \bar{f}_{2}(\xi)=B\left(a-\xi^{\gamma}\right)_{+}^{n_{2}},
$$

where $(b)_{+}=\max (0, b), \quad \gamma=p /(p-1)$, 


$$
n_{1}=\frac{(p-1)\left(p-\left(m_{1}+1\right)\right)}{(p-2)^{2}-\left(m_{1}-1\right)\left(m_{2}-1\right)} ; n_{2}=\frac{(p-1)\left(p-\left(m_{2}+1\right)\right)}{(p-2)^{2}-\left(m_{1}-1\right)\left(m_{2}-1\right)} .
$$

Then in the domain $Q$ according to the comparison principle of solutions [1] [8] we get

Theorem 1. Let $u_{i}(0, x) \leq u_{i \pm}(0, x), x \in R$. Then the solution of the task (1) in the domain $Q$ takes place an estimation

$$
\begin{aligned}
& u_{1}(t, x) \leq u_{1+}(t, x)=\mathrm{e}^{k_{1} t} \tau^{-\alpha_{1}} \bar{f}_{1}(\xi), \\
& u_{2}(t, x) \leq u_{2+}(t, x)=\mathrm{e}^{k_{2} t} \tau^{-\alpha_{2}} \bar{f}_{2}(\xi), \quad \xi=|x| / \tau^{1 / p}
\end{aligned}
$$

where $\bar{f}_{1}(\xi), \bar{f}_{2}(\xi) u \tau(t)$-above-defined functions.

Note that the solution of system (1) when $\beta_{i}=\left[(p-2)^{2}-\left(m_{1}-1\right)\left(m_{2}-1\right)\right] /\left[(p-1)\left(p-\left(m_{i}+1\right)\right)\right]$ has the following representation in the

$$
a=\left[P_{1} \gamma / B\left(\frac{1}{\gamma}, 1+n_{1}\right)\right]^{\frac{\gamma}{n_{1}}}=\left[P_{2} \gamma / B\left(\frac{1}{\gamma}, 1+n_{2}\right)\right]^{\frac{\gamma}{n_{2}}} .
$$

where $B(a, b)$ - Beta Euler function [9].

It is proved that this view is self-similar asymptotics of solutions of systems (1).

$$
\left\{\begin{array}{l}
\tau^{-\frac{1}{\mu_{1}}} \int_{-\infty}^{\infty}\left(a-\xi_{1}^{\gamma}\right)_{+}^{n_{1}} \mathrm{~d} x=P_{1} \\
\tau^{-\frac{1}{\mu_{2}}} \int_{-\infty}^{\infty}\left(a-\xi_{2}^{\gamma}\right)_{+}^{n_{2}} \mathrm{~d} x=P_{2}
\end{array}\right.
$$

Thence

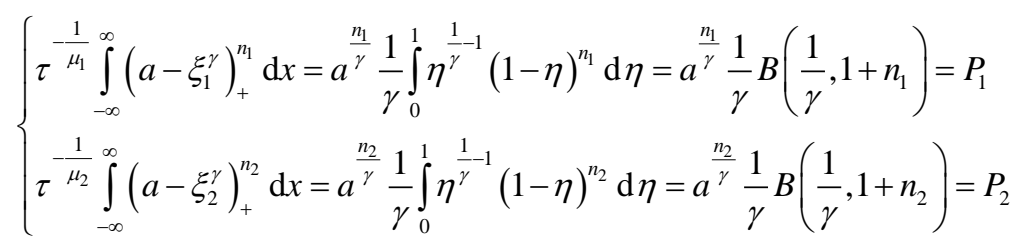

$$
\begin{aligned}
& a=\left[P_{1} \gamma / B\left(\frac{1}{\gamma}, 1+n_{1}\right)\right]^{\frac{\gamma}{n_{1}}}=\left[P_{2} \gamma / B\left(\frac{1}{\gamma}, 1+n_{2}\right)\right]^{\frac{\gamma}{n_{2}}} .
\end{aligned}
$$

Carried out computational experiments and numerical results are obtained (see Table 1, Table 2).

\section{Conclusion}

Thus, it is assumed that the possibility of adequate study of nonlinear equations, biological populations with double nonlinearity based on the method of nonlinear splitting and numerical study of nonlinear processes described by equations with double nonlinearity and analysis of results on the basis of the estimates of the solutions gives a comprehensive picture of the process of multicomponent competing systems of biological populations.

\section{Results}

$$
\left\{\begin{array}{l}
\frac{\partial u}{\partial t}=\frac{\partial}{\partial x}\left(D_{1} v^{m_{1}-1}\left|\frac{\partial u}{\partial x}\right|^{p-2} \frac{\partial u}{\partial x}\right)+k_{1}(t) u\left(1-u^{\beta_{1}}\right), \\
\frac{\partial v}{\partial t}=\frac{\partial}{\partial x}\left(D_{2} u^{m_{2}-1}\left|\frac{\partial v}{\partial x}\right|^{p-2} \frac{\partial v}{\partial x}\right)+k_{2}(t) v\left(1-v^{\beta_{2}}\right)
\end{array}\right.
$$


Table 1. Numerical results in the case of fast diffusion.

\begin{tabular}{|c|c|c|c|c|c|}
\hline Parameter values & $\begin{array}{c}x_{1}=1 ; x_{2}=1 \\
|x|=\sqrt{2}\end{array}$ & $\begin{array}{c}x_{1}=2 ; x_{2}=2 \\
|x|=2 \sqrt{2}\end{array}$ & $\begin{array}{c}x_{1}=3 ; x_{2}=3 \\
|x|=3 \sqrt{2}\end{array}$ & $\begin{array}{c}x_{1}=4 ; x_{2}=4 \\
|x|=4 \sqrt{2}\end{array}$ & $\begin{array}{c}x_{1}=5 ; x_{2}=5 ; \\
|x|=5 \sqrt{2}\end{array}$ \\
\hline
\end{tabular}

$m_{1}=2.5, \quad m_{2}=2.4$,
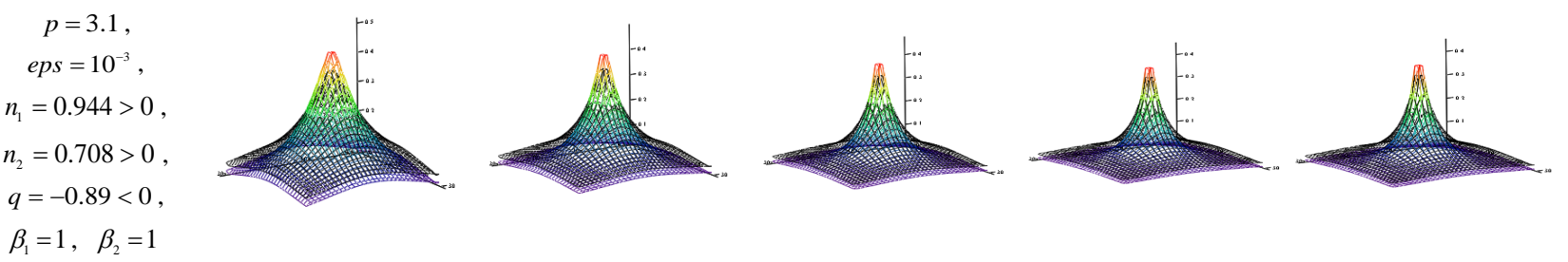

$\beta_{1}=1, \quad \beta_{2}=1$
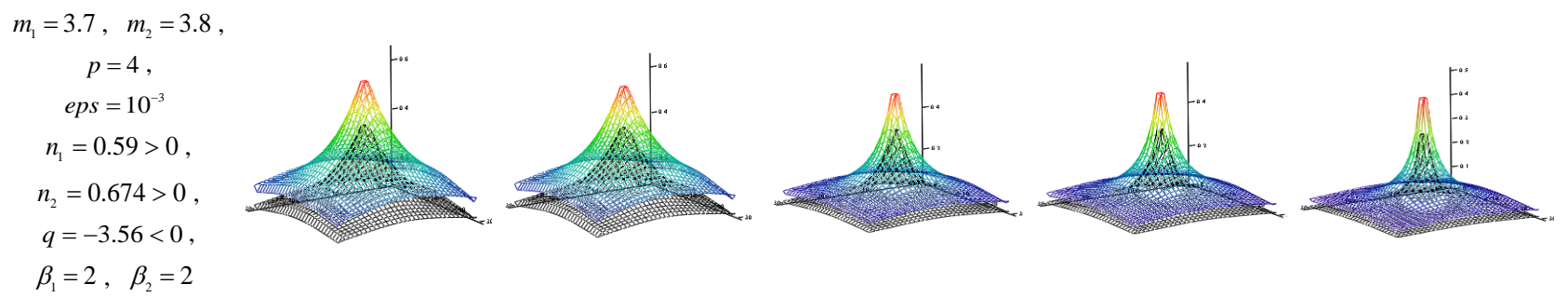

$m_{1}=4.1, \quad m_{2}=4.0$,
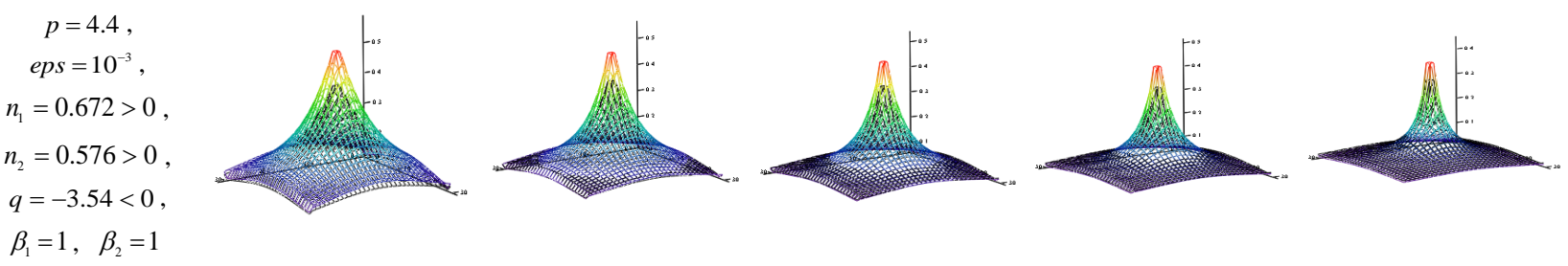

$m_{1}=3.7, \quad m_{2}=3.3$,
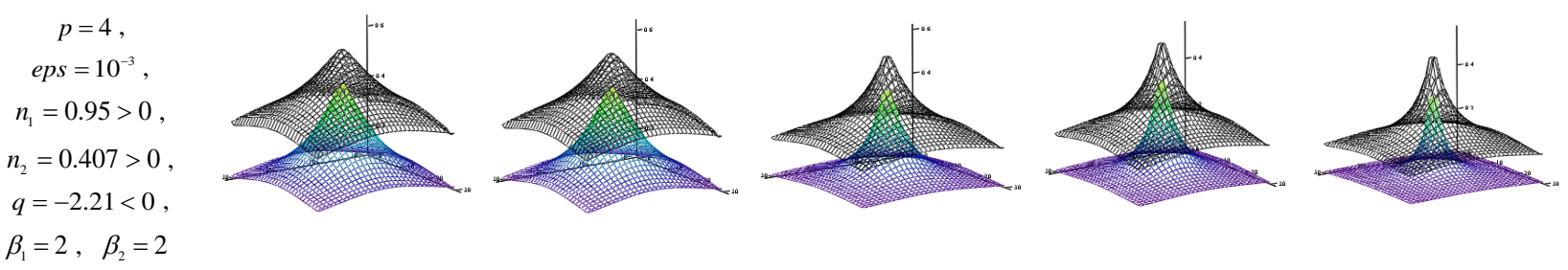

$m_{1}=7, \quad m_{2}=7.1$,

$$
\begin{gathered}
p=4, \\
\text { eps }=10^{-3}, \\
n_{1}=0.368>0, \\
n_{2}=0.377>0, \\
q=-32.6<0, \\
\beta_{1}=3, \quad \beta_{2}=4
\end{gathered}
$$
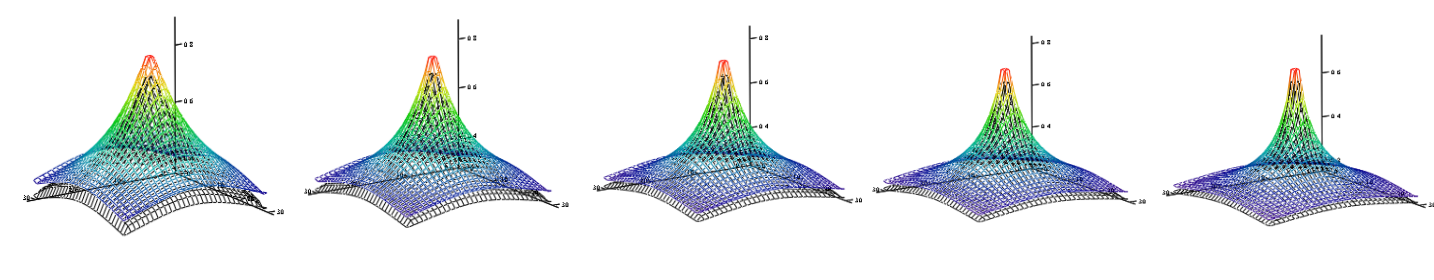
Table 2. Numerical results in the case of slow diffusion.

\begin{tabular}{|c|c|c|c|c|c|}
\hline Parameter values & $\begin{array}{c}x_{1}=1 ; x_{2}=1 \\
|x|=\sqrt{2}\end{array}$ & $\begin{array}{c}x_{1}=2 ; x_{2}=2 \\
|x|=2 \sqrt{2}\end{array}$ & $\begin{array}{c}x_{1}=3 ; x_{2}=3 \\
|x|=3 \sqrt{2}\end{array}$ & $\begin{array}{c}x_{1}=4 ; x_{2}=4 \\
|x|=4 \sqrt{2}\end{array}$ & $\begin{array}{c}x_{1}=5 ; x_{2}=5 ; \\
|x|=5 \sqrt{2}\end{array}$ \\
\hline
\end{tabular}
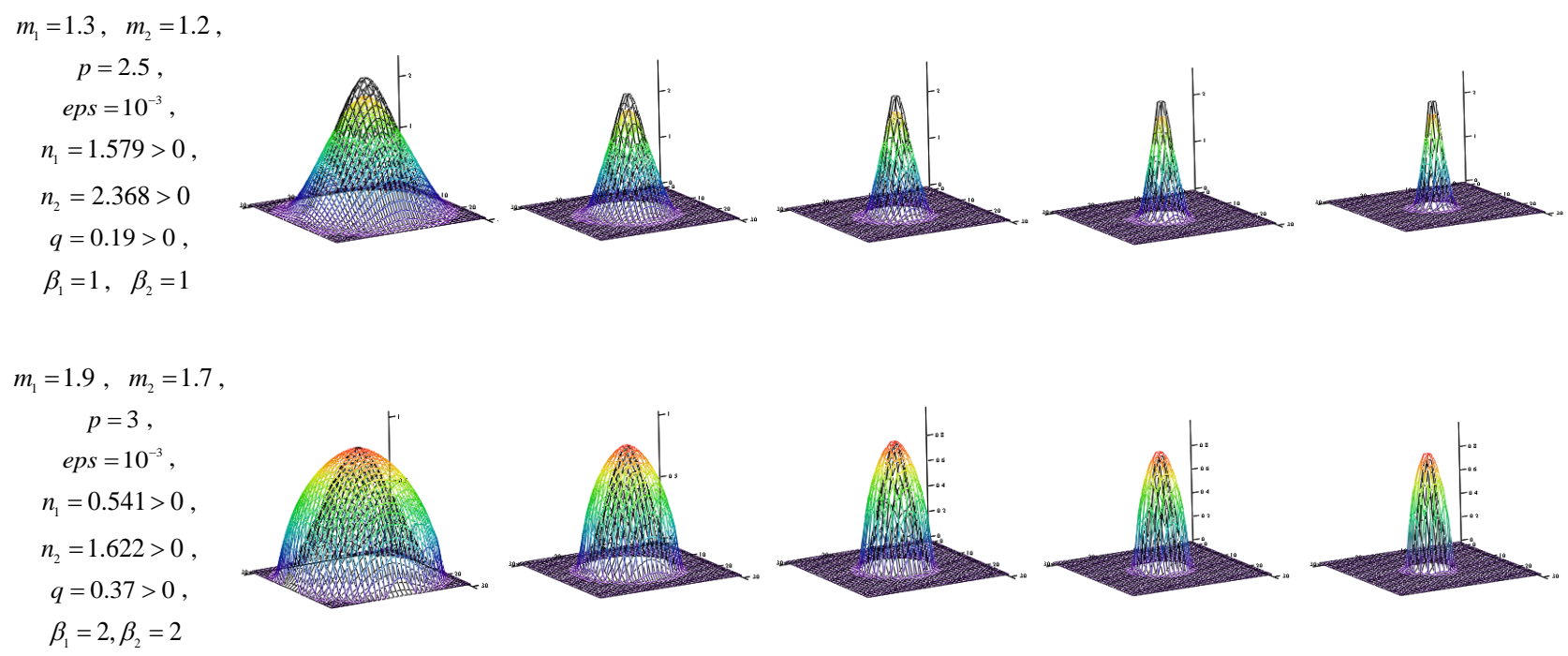

$$
\begin{gathered}
m_{1}=3.7, \quad m_{2}=3.5, \\
p=5.4, \\
\text { eps }=10^{-3}, \\
n_{1}=0.64>0, \\
n_{2}=0.823>0, \\
q=4.81>0, \\
\beta_{1}=3, \quad \beta_{2}=4
\end{gathered}
$$
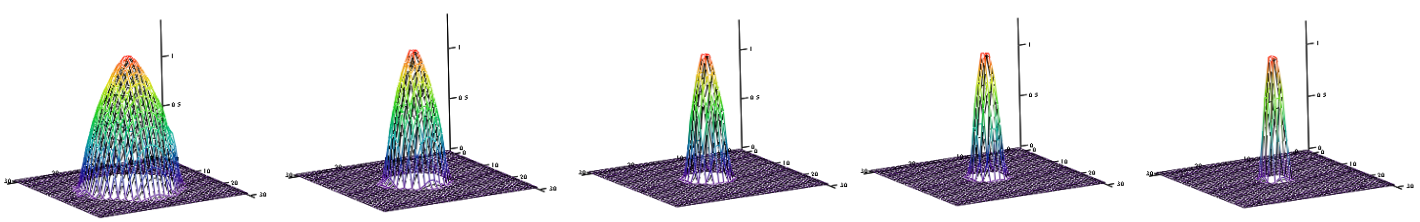

1) Fast diffusion is shown in Table 1. As an initial approximation it is necessary to take:

$$
\begin{gathered}
u_{0}(x, t)=(T+t)^{-\alpha_{1}}\left(a+\xi^{\gamma}\right)^{n_{1}}, v_{0}(x, t)=(T+t)^{-\alpha_{2}}\left(a+\xi^{\gamma}\right)^{n_{2}}, \quad \xi=\frac{|x|}{\tau^{\frac{1}{p}}}, \quad \gamma=\frac{p}{p-1}, \\
n_{i}=\frac{(p-1)\left[p-\left(m_{i}+1\right)\right]}{q}, \quad i=1,2, \quad q=(p-2)^{2}-\left(m_{1}-1\right)\left(m_{2}-1\right)
\end{gathered}
$$

Parameter values must be $n_{1}>0, n_{2}>0, q<0$. Constant $a$ is determined from the condition $\int_{-\infty}^{\infty} u_{1}(x, 0) \mathrm{d} x=P_{1}$,

$$
\int_{-\infty}^{\infty} u_{2}(x, 0) \mathrm{d} x=P_{2}: a=\left(P_{1} \gamma / B\left(\frac{1}{\gamma}, 1+n_{1}\right)\right)^{\frac{\gamma}{n_{1}}}=\left(P_{2} \gamma / B\left(\frac{1}{\gamma}, 1+n_{2}\right)\right)^{\frac{\gamma}{n_{2}}}
$$

2) Slow diffusion is shown in Table 2. As an initial approximation it is necessary to take:

$$
\begin{gathered}
u_{0}(x, t)=(T+t)^{-\alpha_{1}}\left(a-\xi^{\gamma}\right)_{+}^{n_{1}}, \quad v_{0}(x, t)=(T+t)^{-\alpha_{2}}\left(a-\xi^{\gamma}\right)_{+}^{n_{2}}, \quad \xi=\frac{|x|}{\tau^{\frac{1}{p}}}, \\
\gamma=\frac{p}{p-1}, \mathrm{~T}, \quad i=1,2, \quad q=(p-2)^{2}-\left(m_{1}-1\right)\left(m_{2}-1\right)
\end{gathered}
$$


Parameter values must be $n_{1}>0, n_{2}>0, q_{i}>0$. Constant $a$ is determined from the condition

$a=\left(P_{1} \gamma / B\left(\frac{1}{\gamma}, 1+n_{1}\right)\right)^{\frac{\gamma}{n_{1}}}=\left(P_{2} \gamma / B\left(\frac{1}{\gamma}, 1+n_{2}\right)\right)^{\frac{\gamma}{n_{2}}}$.

\section{References}

[1] Aripov, M. (1988) Method Reference Equations for the Solution of Nonlinear Boundary Value Problems. Fan, Tashkent, 137.

[2] Belotelov, N.V. and Lobanov, A.I. (1997) Population Model with Nonlinear Diffusion. Mathematic Modeling, 12, 43-56.

[3] Volterra, V. (1976) The Mathematical Theory of the Struggle for Existence. Science, Moscow, 288.

[4] Gause, G.F. (1934) About the Processes of Destruction of One Species by Another in the Populations of Ciliates. Zoological Journal, 1, 16-27.

[5] Aripov, M. and Muhammadiev, J. (1999) Asymptotic Behaviour of Automodel Solutions for One System of Quasilinear Equations of Parabolic Type. France. Buletin Stiintific-Universitatea din Pitesti, Seria Matematica si Informatica, 19-40.

[6] Aripov, M.M. and Muhamediyeva, D.K. (2013) To the Numerical Modeling of Self-Similar Solutions of ReactionDiffusion System of the One Task of Biological Population of Kolmogorov-Fisher Type. International Journal of Engineering and Technology, 2, 281-286.

[7] Aripov, M.M. and Muhamedieva, D.K. (2013) Approaches to the Solution of One Problem of Biological Populations. Issues of Computational and Applied Mathematics, 129, 22-31.

[8] Murray, D.J. (1983) Nonlinear Diffusion Equations in Biology. Mir, Moscow, 397.

[9] Huashui, Z. (2010) The Asymptotic Behavior of Solutions for a Class of Doubly Degenerate Nonlinear Parabolic Equations. Journal of Mathematical Analysis and Applications, 370, 1-10. http://dx.doi.org/10.1016/j.jmaa.2010.05.003 